\title{
Tecnologia assistiva no contexto do PARFOR da Universidade Federal do Maranhão (UFMA)
}

\section{Assistive technology in the context of PARFOR of the Federal University of Maranhão (UFMA)}

\author{
Cenidalva Miranda de Sousa Teixeira ${ }^{1 *}$, Raimunda Ramos Marinho ${ }^{1}$, Jennyfer de O.
}

Diniz 1

\begin{abstract}
RESUMO
Este trabalho consiste em investigar a presença da Tecnologia Assistiva como recurso de apoio pedagógico no processo de formação de professores com vistas a perceber o grau de conhecimento e reflexão sobre a temática no percurso formativo dos professores no contexto de um programa de formação de professores para educação básica - PARFOR. Como metodologia utilizou-se pesquisas bibliográfica, documental e de campo. O universo da pesquisa foram os cursos ofertados pelo PARFOR na Universidade Federal do Maranhão, a partir da análise de projetos pedagógicos e suas disciplinas instrumentais. A amostragem contou com quatro cursos que correspondem a 50\% do universo. Na pesquisa documental, observou-se a ausência dos termos 'acessibilidade' e 'tecnologia assistiva' nas ementas das disciplinas instrumentais dos cursos ofertados. Conclui-se que a ausência desses tópicos nas ementas dos cursos ofertados dificulta a compreensão social e a promoção da acessibilidade.
\end{abstract}

Palavras-chave: Acessibilidade; Inclusão social; Tecnologia assistiva; PARFOR.

\begin{abstract}
This paper consists of investigating the presence of Assistive Technology as a resource for pedagogical support in the process of teacher education in order to understand the degree of knowledge and reflection on the subject in the training path of teachers in the context of a teacher training program for education basic - PARFOR. The methodology used was bibliographical, documental and field research. The research universe was the courses offered by PARFOR at the Federal University of Maranhão, based on the analysis of pedagogical projects and their instrumental disciplines. The sample consisted of four courses that correspond to $50 \%$ of the universe. In the documental research, the absence of the terms 'accessibility' and 'assistive technology' was observed in the menus of the instrumental disciplines of the courses offered. It is concluded that the absence of these topics in the menus of the courses offered makes social understanding and the promotion of accessibility difficult.
\end{abstract}

Keywords: Accessibility; Social inclusion; Assistive technology; PARFOR.]

${ }^{1}$ Universidade Federal do Maranhão. cenidalva@gmail.com; rr.marinho@ufma.br ; jennyfer.od@discente.ufma.br 


\section{INTRODUÇÃO}

As discussões a respeito da Tecnologia Assistiva (TA) são de suma importância para a desconstrução da ideia de deficiência como sinônimo de incapacidade, pois as pessoas com deficiência podem não desempenhar algumas tarefas devido às barreiras do ambiente no qual estão inseridas, e não por serem incapazes.

Nesse sentido, pesquisas nesse campo contribuem para o desenvolvimento de novos recursos tecnológicos destinados à acessibilidade, uma vez que a tecnologia consiste na aplicação dos conhecimentos científicos produzidos. De modo positivo, essas discussões despertam uma maior consciência social nas pessoas. Teixeira, Ferreira e Vetter (2020) enfatizam que a pesquisa na área se torna relevante por proporcionar um espaço de reflexão sobre a usabilidade desse recurso no processo de mediação pedagógica e aprendizagem, tendo em vista uma realidade educacional e socialmente construída para a formação do cidadão.

Os autores complementam que a aquisição de habilidades referentes ao uso desses recursos acessíveis e gratuitos é possível por meio da prática, tanto de estudantes quanto de professores, orientada por manuais e tutoriais disponíveis na internet. Outro ponto de destaque no que diz respeito à presença da Tecnologia Assistiva nas salas de aula das Instituições de Ensino Superior é o incentivo ao desenvolvimento de pesquisas sobre a temática.

Este estudo se constitui em uma investigação de cunho qualitativo, cujas discussões epistemológicas e metodológicas estão baseadas em dados empíricoanalíticos. Esses dados podem apresentar o impacto, a difusão e a inserção da tecnologia assistiva na estrutura curricular e processos formativos de professores da educação básica, ou, melhor, o objeto de estudo é a Tecnologia Assistiva - TA e seu uso como ferramenta na mediação pedagógica para a aprendizagem do aluno com deficiência.

Apresentamos, portanto, a possibilidade de utilização da TA no cotidiano da escola, seja ela regular ou especial, buscando oferecer condições de atender às necessidades de conhecimento e reflexão sobre essa temática, e ampliar a discussão sobre os benefícios de investir na tecnologia como apoio pedagógico e oportunidade de participar e interagir com o meio através dos recursos que a mesma oferece.

Assim, o objetivo geral é investigar a presença da Tecnologia Assistiva como recurso de apoio pedagógico no processo de formação de professores com vistas a 
perceber o grau de conhecimento e reflexão sobre a temática no percurso formativo dos professores no contexto de um programa de formação de professores para educação básica - PARFOR. Quanto aos objetivos específicos, temos: a) identificar os principais conceitos relacionados à acessibilidade e à tecnologia assistiva no campo socioeducacional; b) conhecer as ferramentas de tecnologia assistiva disponíveis no mercado para pessoas com deficiência visual, auditiva, intelectual e motora; d) refletir sobre a tecnologia assistiva no contexto da formação de professores da educação básica - PARFOR; e) analisar no contexto curricular dos cursos ofertados pelo PARFOR/UFMA a presença da tecnologia assistiva nas disciplinas instrumentais. A metodologia utilizada está descrita na seção 3.

\section{REFERENCIAL TEÓRICO}

As primeiras discussões sobre acessibilidade datam da década de 1970, após a criação da Lei de Reabilitação (Rehabilitation Act) dos Estados Unidos, sendo o auge de tais discussões a década de 1980 com a criação da ADA (Americans with Disabilities Act). Outro fato importante para a área foi a publicação, em 1993, pela Organização das Nações Unidas (ONU), das Normas sobre a Igualdade de Oportunidades para as Pessoas com Deficiência (FROTA, 2013).

No Brasil, as discussões acadêmicas referentes ao tema acessibilidade remontam à década de 1990 (WAGNER et al., 2010), mas alguns debates já eram realizados na década anterior. Em 1985, a Associação Brasileira de Normas Técnicas (ABNT) publicou a NBR 9050, estabelecendo critérios para construção e adaptação de edificações, mobiliário, espaços e equipamentos urbanos. Outro marco normativo foi o Decreto-Lei $n^{\circ}$ 6.494/2009, que promulgou a Convenção Nacional sobre o Direito das Pessoas com Deficiência.

A acessibilidade é uma área de estudo em crescimento no país, mas existe ainda um longo caminho a ser percorrido para que a mesma se consolide, de modo a desconstruir os estereótipos arraigados no imaginário popular, como, por exemplo, a visão na qual a acessibilidade consiste no acesso físico de cadeirantes possibilitado pelas rampas. Existem outros fatores que envolvem a acessibilidade, o que pode ser percebido no conceito apresentado na legislação brasileira, pela qual a acessibilidade é compreendida como:

[...] possibilidade e condição de alcance para utilização, com segurança e autonomia, de espaços, mobiliários, equipamentos 
urbanos, edificações, transportes, informação e comunicação, inclusive seus sistemas e tecnologias, bem como de outros serviços e instalações abertos ao público, de uso público ou privados de uso coletivo, tanto na zona urbana como na rural, por pessoa com deficiência ou com mobilidade reduzida (BRASIL, 2000, p. 2).

O uso de tecnologias na educação de alunos por professores vem aumentando a cada dia, considerando que elas "[...] prometem desempenhar um papel significativo no desenvolvimento de competências e habilidades dos professores e alunos" (BOTTENTUIT JUNIOR, 2010, p. 28). As ferramentas tecnológicas podem ser utilizadas no processo de ensino-aprendizagem e, consequentemente, na sala de aula, de acordo com a sua aplicabilidade, como ressalta Teixeira e Carvalho (2020). Para complementar, Silva e Teixeira (2020) destacam que elas assumem um papel essencial na formação de professores, potencializando suas práticas e favorecendo novas habilidades e competências. Nesse cenário, tem-se a TA que vem sendo utilizada com o mesmo propósito.

Na educação, o uso da TA auxilia o processo de ensino-aprendizagem. A TA pode ser vista como uma ferramenta na mediação pedagógica para a aprendizagem do aluno com deficiência. Nesse aspecto, Bersch (2006, p. 92) destaca que "a aplicação da Tecnologia Assistiva na educação vai além de simplesmente auxiliar o aluno a fazer tarefas pretendidas. Nela, encontramos meios de o aluno ser e atuar de forma construtiva no seu processo de desenvolvimento".

Assim, esta pesquisa busca verificar a inserção da TA nos currículos dos cursos ofertados pelo PARFOR/UFMA, com vistas a entender a relevância e contribuição efetiva para o processo de formação de professores e gestores da educação básica.

Percebemos que a aplicabilidade da Tecnologia Assistiva requer a análise de necessidades dos/as educando/as, tornando imprescindível a formação dos/as professores/as para o conhecimento de metodologias, estratégias e aplicação de recursos tecnológicos que auxiliem e facilitem o processo de ensino-aprendizagem. Para a área da Educação, especificamente, este estudo contribui para o fortalecimento do lado humanístico e social da área, favorecendo a busca de conhecimentos e reflexões quanto à importância de sua capacitação, a fim de compreender as facilidades que a Tecnologia Assistiva permite aos seus usuários.

Essas pesquisas são relevantes por justificarem a importância da Tecnologia Assistiva e por caracterizarem a utilização destas no dia a dia das pessoas com 
deficiência, reforçando os argumentos relacionados ao cumprimento dos direitos desses cidadãos (VARELA; OLIVER, 2012). As pesquisas contribuem ainda para o combate de práticas discriminatórias através da conscientização social. Observamos a pertinência do tema no processo de formação de profissionais dos diversos ramos do conhecimento, não ficando restrito aos campos de Terapia Ocupacional e Educação Inclusiva.

\section{METODOLOGIA}

O estudo teve caráter exploratório, pois buscou familiarização com o problema para esclarecê-lo (GIL, 2002), e descritivo, por ter se focalizado na descrição das características do objeto de pesquisa (BARROS; LEHFELD, 2007).

O primeiro passo para a realização da pesquisa foi a identificação de fontes de informação que tratavam do tema para embasamento teórico. A busca foi feita na internet por meio do buscador Google, sendo usadas as seguintes palavras-chave: acessibilidade, tecnologias acessíveis, Tecnologia Assistiva, educação especial, educação inclusiva etc.

Após a identificação das fontes, fizemos a pré-leitura das obras para selecionar os documentos que poderiam compor a fundamentação do estudo. Foram examinados a folha de rosto, o sumário, os índices, as referências bibliográficas, as notas ao pé da página, o prefácio, a introdução e a conclusão (CERVO; BERVIAN; SILVA, 2007).

O passo seguinte foi a leitura seletiva, com o olhar voltado ao problema formulado e às perguntas, elaboradas quando se questionava o assunto. Foram identificadas e selecionadas informações que pudessem fornecer esclarecimentos sobre o problema (CERVO; BERVIAN; SILVA, 2007). Fizemos a identificação dos principais autores que investigam o tema Tecnologia Assistiva, das principais discussões relacionadas a esse campo e do papel na inclusão de pessoas com deficiência no Ensino Superior para nortear a análise dos resultados encontrados.

Todos esses passos compuseram a pesquisa bibliográfica. De acordo com Marconi e Lakatos (2003, p. 183), esse tipo de pesquisa tem por objetivo “[...] colocar o pesquisador em contato direto com tudo o que foi escrito, dito ou filmado sobre determinado assunto [...]".

Este estudo incluiu ainda pesquisa documental, na qual foram analisados documentos como o Projeto Pedagógico dos cursos presentes no PARFOR, a fim de obter informações que contribuíssem para a construção do instrumento de coleta de 
dados e compreensão do objeto a partir de dispositivo institucional, o qual norteia a formação, a citar o currículo.

Após a seleção dos documentos nas pesquisas bibliográfica e documental, foram feitas as leituras reflexiva e interpretativa. Na leitura reflexiva foram identificadas ideias principais e secundárias, determinando-se, com base nos objetivos da pesquisa, sua utilidade (CERVO; BERVIAN; SILVA, 2007). Na leitura interpretativa, as ideias identificadas anteriormente foram aplicadas para fins específicos do estudo, embasando as ideias do pesquisador.

O próximo passo foi a coleta de dados que, segundo Barros e Lehfeld (2007), Cervo, Bervian e Silva (2007), consiste na fase em que a realidade é indagada. Por meio da aplicação de técnicas, os dados são obtidos. Nessa fase foram determinados a população do estudo, o instrumento de coleta de dados e a sua aplicação.

Na pesquisa de campo, os sujeitos envolvidos foram os professores da disciplina Libras que ministraram aula nos Programa de Formação de Professores para Educação Básica - PARFOR, em particular nos cursos que foram mapeados na pesquisa documental. Apresentamos, então, as etapas de acordo com os objetivos propostos:

$\mathrm{Na}$ etapa 1, o objetivo foi identificar os principais conceitos relacionados à acessibilidade e à tecnologia assistiva no campo socioeducacional. Para a sua realização foi feita pesquisa bibliográfica e documental, ocasião em que foram levantados os autores Masini (2007), Bersch (2006), Varela e Oliver (2012), Wagner (2010), Rodrigues e Alves (2013), Bottentuit Junior (2010), Teixeira e Carvalho (2020), dentre outros. Esses autores auxiliaram na familiarização do tema, elaboração da contextualização e fundamentação teórica da pesquisa.

A etapa 2 teve como objetivo identificar os cursos de formação de professores ofertados pelo PARFOR/UFMA e seus respectivos coordenadores, por meio da pesquisa documental. Essa etapa foi cumprida em maio de 2020 e teve como resultado a identificação dos seguintes cursos dentro do projeto: Matemática, Educação Física, Letras Inglês, Letras Espanhol, História, Geografia, Filosofia e Pedagogia. Foram identificados oito cursos ofertados. Destes, conseguimos trabalhar com quatro (4). O passo seguinte foi realizar um levantamento das disciplinas instrumentais constantes no Projeto Pedagógico dos cursos estudados.

A etapa 3 consistiu na identificação dos softwares de tecnologia assistiva disponíveis no mercado para pessoas com deficiência visual, auditiva e motora. A 
identificação ocorreu por meio de pesquisa bibliográfica, documental e em sites especializados, destinados ao mapeamento. Foram realizadas pesquisas com o objetivo de identificar os softwares no mercado para cada deficiência acima citada.

$\mathrm{Na}$ etapa 4 identificamos as disciplinas que tratam sobre acessibilidade e tecnologia assistiva nos currículos via projetos pedagógicos dos cursos ofertados no PARFOR/UFMA. A pesquisa documental atendeu ao objetivo específico que foi analisar no contexto curricular dos cursos ofertados pelo PARFOR/UFMA a presença da tecnologia assistiva nas disciplinas instrumentais. Nessa etapa, observamos que cada curso apresentava três disciplinas instrumentais com nomenclaturas diferentes. Essas disciplinas abordavam sobre tecnologia, porém a disciplina Libras mantinha a mesma nomenclatura em todos os cursos do PARFOR.

Por esse motivo, elaboramos a pesquisa de campo com os professores que ministraram a disciplina Libras no âmbito do PARFOR.

\section{APRESENTAÇÃO E DISCUSSÃO DOS RESULTADOS}

Como resultado, no primeiro momento foram realizadas a análise dos Projetos Pedagógicos enviados pelos coordenadores de quatro (4) dos oito (8) cursos presentes no PARFOR. Foram observados aspectos como objetivo e metodologia do curso, além das ementas, bibliografia básica e complementar das disciplinas instrumentais que compõem o curso.

Nos quatro (4) cursos analisados foi possível observar a existência da disciplina Tecnologias da Informação e Comunicação, mas em nenhuma delas consta em sua ementa os termos 'acessibilidade' e 'tecnologia assistiva'. Na disciplina Educação Especial, também presente nos quatros cursos analisados, observamos que a ementa traz as palavras: paradigmas sociais e inclusão de alunos com deficiência. Contudo, a ementa não contempla os termos 'acessibilidade' e 'tecnologia assistiva'. Nessas duas disciplinas a carga horária foi de sessenta horas (60h).

Além dessas disciplinas, o curso igualmente dispõe da disciplina de Língua Brasileira de Sinais (LIBRAS), cuja carga horária também é de sessenta (60) horas. Esta última é ofertada em todos os cursos no âmbito do PARFOR com a mesma nomenclatura. Mesmo não contemplando os termos 'acessibilidade' e 'tecnologia assistiva' na sua ementa, optamos por realizar a pesquisa de campo com os professores que ministram essa disciplina no âmbito do PARFOR. 
Com base no exposto, afirmamos sobre a ausência dos termos 'acessibilidade' e 'tecnologia assistiva' nas ementas das disciplinas instrumentais ministradas nos cursos ofertados no âmbito do PARFOR. Com base nesses dados, foi elaborado um questionário e enviado aos professores que ministraram a disciplina Libras em todos os cursos ofertados no âmbito do PARFOR. Consideramos que essa disciplina está diretamente relacionada aos termos 'acessibilidade' e 'tecnologia assistiva'.

No questionário foram consideradas questões como a abordagem, nível de conhecimento e utilização da TA nas aulas da disciplina Libras, em cursos ofertados no âmbito do PARFOR. O questionário foi disponibilizado através da plataforma Google Forms e direcionado a seis professores de Libras dos cursos. Apenas quatro (4) professores retornaram com os resultados. Logo, o universo da pesquisa foi constituído por 6 professores e a amostragem por quatro, o que compreende, aproximadamente, a $67 \%$ do universo.

A análise dos resultados do questionário será apresentada de acordo com a ordem dos enunciados.

Entre os quatorze (14) softwares levantados na pesquisa, apenas quatro foram marcados como conhecidos, entre eles Liane TTS e Rybená, com 25\% cada. Já os softwares como Braille Translator e Surdo Bilíngue foram marcados por $50 \%$ dos participantes da pesquisa. Ao serem questionados sobre outros softwares além dos levantados, os participantes adicionaram o Hand Talk, Dicionário Digital e TV Libras.

No que se refere à experiência com alunos com deficiência dentro do PARFOR, $75 \%$ dos participantes já ensinaram alunos com alguma deficiência e 25\% não tiveram essa experiência. Entre as deficiências abordadas no questionário, foi citada a visual.

Quando o assunto foi a abordagem do tema TA durante a formação dos professores de Libras, $50 \%$ afirmaram que tiveram contato com o tema. Dentre os que disseram sim, houve comentários relacionados ao ensino $\mathrm{EaD}$ e à facilidade de demonstração de softwares, uma vez que os formandos estão lidando diretamente com tecnologia. Quanto aos professores que disseram que não aprenderam sobre TA na formação de Libras, foi ressaltado que houve contatos com a TA durante a formação continuada em cursos de especialização e outros cursos de curta duração. Sobre esse aspecto, Mercado (1998) destaca que o ensino continuado permitiu aos professores uma melhor contextualização e propriedade a respeito de fatores que a graduação não cobriu ou se aprofundou. 
Quando questionados sobre as outras ferramentas assistivas que eram conhecidas e/ou utilizadas nas aulas teóricas e/ou práticas por esses professores, alguns citaram o SIGAA, a plataforma online das Universidades Federais, vídeos, glossários, App de Libras, canais do YouTube, dicionário digital, estudo de metodologias ativas, alguns aplicativos não exemplificados e também sites para a propagação do ensino de Libras em grupo de estudos.

Observamos que 50\% dos professores responderam que tiveram oportunidade de fazer uso de algum tipo de tecnologia assistiva durante a ministração de disciplinas no PARFOR; os outros 50\% utilizaram apenas como projeto teórico. Entre a TA utilizada, estavam inclusos: aplicativos não exemplificados, SIGAA, vídeos, glossários, Vlibras, App de Libras, canais do YouTube, Hand Talk, vídeos de Libras no YouTube e Dicionário Digital do Instituto Nacional de Educação de Surdos. Nesse cenário, observamos que o uso das novas tecnologias por professores e alunos vem aumentando de forma gradativa nas salas de aula. Esse dado já foi apontado anteriormente pelos autores Silva e Teixeira (2020) e Teixeira e Carvalho (2020).

Também foi questionado se os participantes da pesquisa achavam importante que na ementa da disciplina fosse apresentado o assunto relacionado à Tecnologia Assistiva. Todos (100\%) responderam que sim. Quando comentaram sobre a afirmação, um dos participantes replicou que "Com o advento da Tecnologia é essencial que os docentes e os graduandos como futuros professores, tenham acesso às novas tecnologias".

Outros participantes comentaram sobre o papel da TA, como o auxílio na metodologia aplicada ao processo do ensino de Libras. Outro professor respondeu que a inclusão do assunto TA nas ementas da disciplina de Libras do PARFOR é importante, pois a TA é uma ferramenta que visa proporcionar autonomia para as pessoas com deficiência auditiva e surdos. Logo, é relevante que os graduandos conheçam, além do desenho universal, o direito ao acesso e à acessibilidade, independente de idade, contexto socioeconômico e cultura.

Convém ressaltar que a experiência e o processo de desenvolvimento da pessoa com deficiência e limitações são diferentes para cada sujeito, já que cada um tem sua individualidade. Masini (2007) enfatiza que é necessário que haja um universo extenso de softwares, uma vez que quanto mais opções disponíveis, maior será o número de pessoas saciadas. 
A TA compensa dificuldades e auxilia com instrumentos de adaptação nas limitações enfrentadas por pessoas com deficiências, a fim de aumentar sua autonomia em procedimentos e tomadas de decisão. Essas ajudas técnicas, segundo o Decreto $\mathrm{n}^{\circ}$ 3.298/1999, são “elementos que permitem compensar uma ou mais limitações funcionais motoras, sensoriais ou mentais da pessoa portadora de deficiência, com o objetivo de superar as barreiras de comunicação e da mobilidade e de possibilitar sua plena inclusão social" (CEDIPOD, 2007, não paginado).

\section{CONSIDERAÇÕES FINAIS}

Durante a fase de pesquisa bibliográfica, identificamos o conceito de Tecnologia Assistiva como uma área de conhecimento interdisciplinar e indispensável à construção de uma sociedade inclusiva, cuja participação efetiva de todos os cidadãos é possibilitada pela equiparação de oportunidades.

Além disso, verificamos o papel dessas tecnologias no contexto acadêmico, consideradas instrumentos de apoio ao estudante com deficiência, como tema de discussões e pesquisas, e como conteúdo necessário à formação completa de profissionais em todas as áreas de conhecimento. A fase de pesquisa documental foi importante para o mapeamento dos cursos ofertados pelo PARFOR/UFMA e para a reunião de informações destinadas à construção do instrumento de coleta de dados e ao prosseguimento das demais etapas deste estudo.

A fase de pesquisa empírica proporcionou a obtenção de um panorama relacionado à utilização e conhecimento no que diz respeito à Tecnologia Assistiva nos cursos ofertados pelo PARFOR na UFMA. Contudo, no aspecto geral, percebemos que há um consenso sobre a importância da TA na inclusão das pessoas com deficiência em todos os âmbitos sociais.

Assim, esta pesquisa possibilitou a compreensão da amplitude do campo da Tecnologia Assistiva, notando-se lacunas e carências na área.

\section{REFERÊNCIAS}

BARROS, Aidil Jesus da Silveira; LEHFELD, Neide Aparecida de Souza. A pesquisa e a iniciação científicas. In: . Fundamentos de Metodologia Científica. 3. ed.

São Paulo: Pearson Prentice Hall, 2007. cap. 7, p. 105-118.

BERSCH, R. Tecnologia assistiva e educação inclusiva. In: Ensaios Pedagógicos, Brasília: SEESP/MEC, p. 89-94, 2006. 
BOTTENTUIT JUNIOR, J. B. Concepção, Avaliação e Dinamização de um Portal Educacional de WebQuests em Língua Portuguesa. 2010. 637f. Tese (Doutoramento em Ciências da Educação, Área de Conhecimento de Tecnologia Educativa) Universidade do Minho, Instituto de Educação, Braga, 2010. Disponível em: https://repositorium.sdum.uminho.pt/bitstream/1822/11889/1/tese.pdf. Acesso em: 28 maio 2020

BRASIL. Lei $\mathrm{n}^{\circ} 10.098$, de 19 de dezembro de 2000. Estabelece normas gerais e critérios básicos para a promoção da acessibilidade das pessoas portadoras de deficiência ou com mobilidade reduzida, e dá outras providências. Diário Oficial da União, Brasília, DF, 20 dez. 2000. Disponível em: $<$ https://www.jusbrasil.com.br/diarios/1542695/pg-2-secao-1-diario-oficial-da-uniaodou-de-20-12-2000?ref=next_button>. Acesso em: 12 out. 2017.

CEDIPOD. 1999. Legislação. Decreto no 3.298 de 22/12/1999. Disponível em: http://www.cedipod.org.br/dec3298.htm. Acesso em: 10 dez. 2007.

CERVO, Amado Luiz; BERVIAN, Pedro Alcino; SILVA, Roberto da. Elaboração e comunicação da pesquisa. In: Pearson Prentice Hall, 2007. cap. 6, p. 71-90. Metodologia Científica. 6. ed. São Paulo:

FROTA, Taís. Acessibilidade: o início. Acessibiteca, 2013. Disponível em: <http://www.acessibiteca.uff.br/?page_id=724>. Acesso em: 12 out. 2017.

GIL, Antônio Carlos. Como classificar as pesquisas? In: Projeto de Pesquisa. 4. ed. São Paulo: Atlas, 2002. cap.4, p. 41-58.

MARCONI, Marina de Andrade; LAKATOS, Eva Maria. Técnicas de Pesquisa. In:__ Fundamentos de Metodologia Científica. 5. ed. São Paulo, Atlas: 2003. cap. 9, p. 174-214.

MASINI, E. A. F. S. A pessoa com deficiência visual: um livro para educadores. São Paulo, SP: Vetor, 2007, 262 p.

MERCADO, Luís P. L. Formação docente e novas tecnologias. In: MERCADO, Luís P. L. (Org.). Novas tecnologias na educação: reflexões sobre a prática. Maceió: EDUFAL, 2002. p.11-28.

RODRIGUES, Patrícia Rocha; ALVES, Lynn Rosalina Gama. Tecnologia Assistiva: uma revisão do tema. Holos, ano 29, v. 26, p. 170-180, 2013. Disponível em: http://200.9.65.226/bitstream/fieb/687/1/Tecnologia\%20assistiva\%20\%20uma\%20revis\%c3\%a3o\%20....pdf. Acesso em: 21 set. 2020.

SILVA, Chayene Cristina Santos Carvalho; TEIXEIRA, Cenidalva M. de S. O uso das tecnologias na educação: os desafios frente à pandemia da COVID-19.

Brazilian Journal of Development, v. 6, n. 9 , p. 2020. DOI:10.34117/bjdv6n9452

TEIXEIRA, Cenidalva M. de S.; FERREIRA, Vanessa T.F.; VETTER, Silvana M. J. Tecnologia Assistiva nos cursos de Biblioteconomia das Instituições de Ensino Superior públicas das regiões Norte e Nordeste do Brasil. In: SIMPÓSIO INTERNACIONAL E 
NACIONAL DE TECNOLOGIAS DIGITAIS NA EDUCAÇÃO, 5., 2020, São Luís. Anais eletrônicos [...]. São Luís: EDUFMA, 2020. p. 2538-2547.

TEIXEIRA, Cenidalva; CARVALHO, S. M. A gamificação como prática de ensino na disciplina Automação de Unidades de Informação. Revista Querubim (Online), v. 16, p. 20-25, 2020.

VARELA, Renata Cristina Bertolozzi; OLIVER, Fátima Corrêa. A utilização de Tecnologia Assistiva na vida cotidiana de crianças com deficiência. 2012. Disponível em: https://www.scielosp.org/article/csc/2013.v18n6/1773-1784/pt/. Acesso em: 25 set. 2020.

WAGNER, et al. Acessibilidade de pessoas com deficiência: um olhar da comunidade da periferia de Porto Alegre. Ciência em Movimento, ano 7, n. 23, 2010/1. Disponível em: https://www.metodista.br/revistas/revistas-ipa/index.php/RS/article/viewFile/94/58. Acesso em: 17 set. 2020. 